\title{
Upper gastrointestinal tract involvement in the management of bariatric patients in the Kingdom of Saudi Arabia
}

\author{
Georgios Zacharakisa, Abdulaziz Almasoud b, Jamaan AlZahranic, Sameer Al-Ghamdic, \\ Abdullah Altuwaim a , Abdullah AlShehrid , Abdullah Bawazira, Ahmad Alonazia , Faisal Alsamaria, \\ Mohammed Alajmi ${ }^{\text {, Ahmed Lotfy }}{ }^{e}$, Alexandros Kyritsis ${ }^{e}$, Pavlos Nikolaidisf, loannis Terzise \\ Prince Sattam bin Abdulaziz University Hospital College of Medicine, Al Kharj; Prince Sultan Military Medical \\ City, Riyadh; Military Hospital, Al Kharj; Prince Sattam bin Abdulaziz University College of Medicine, Al Kharj; \\ King Salman Specialist Hospital, Hail, Al-Imam Mohammad Ibn Saud Islamic University College of Computer and \\ Information Sciences, Riyadh, Saudi Arabia
}

\section{Abstract}

\section{Conflict of Interest: None}

Correspondence to: Georgios Zacharakis, Head of Endoscopy Unit, Department of Internal Medicine, Prince Sattam bin Abdulaziz University Hospital, College of Medicine, Prince Sattam bin Abdulaziz University, Prince Sattam bin Abdulaziz Road, Ar Rayyan, Al Kharj, 16277, Saudi Arabia, e-mail: GZacharakis@yahoo.gr

Received 21 July 2020; accepted 22 October 2020; published online 16 January 2021

DOI: https://doi.org/10.20524/aog.2021.0576
Background Preoperative esophagogastroduodenoscopy (EGD) may affect the management of bariatric patients although this is not consistent universally. The present prospective study evaluated the effect of preoperative EGD findings in obese Saudi patients, including upper digestive symptoms (UDS) and comorbidities, on their planned surgery.

Methods From January 2018 to May 2019, we conducted a 4-center retrospective observational study to evaluate the endoscopic findings among Saudi patients aged 18-65 years with a body mass index $(\mathrm{BMI})>40 \mathrm{~kg} / \mathrm{m}^{2}$. Preoperative data included UDS, comorbidities, Helicobacter pylori (H. pylori) infection assessed during a histopathological examination, and EGD findings.

Results 717 patients underwent EGDs, and 432 underwent bariatric surgery. The mean BMI was $44.3 \pm 6.3 \mathrm{~kg} / \mathrm{m}^{2}$, and the mean age was $27.8 \pm 11.8$ years. The overall UDS prevalence was $49 \%$, with the most frequent being gastroesophageal reflux disease 54\% (387/717), followed by dyspepsia $44 \%$ (315/717). H. pylori infection was detected in 287/672 (42.4\%) patients. The total percentage of patients with normal EGD was $36 \%(258 / 717)$. A delayed bariatric procedure was performed in $15 \%$ of the patients for the following reasons: $2.3 \%$ had large polyps of $>1 \mathrm{~cm}$ (either hyperplastic or cystic polyps); $1.62 \%$ had esophagitis grade $\mathrm{C}$ and D based on the Los Angeles classification; $0.7 \%$ had Barrett's esophagus; and $5.7 \%$ had peptic ulcer disease.

Conclusions Our findings confirmed that obesity carries a profound health burden with a significant impact on health expenditures. Routine preoperative EGD in the obese Saudi population appears to be mandatory to identify factors that may change, delay, or postpone the bariatric procedure.

Keywords Bariatric surgery, morbid obesity, Helicobacter pylori, endoscopic findings, delayed or postponed surgery

Ann Gastroenterol 2021; 35 (2): 1-6

\section{Introduction}

In Saudi Arabia, obesity is a serious problem with an increasing prevalence; $30 \%$ of men and $44 \%$ of women are obese. Saudi Arabia has the 11th highest adult obesity rate among 188 countries [1]. Laparoscopic sleeve gastrectomy (LSG) and laparoscopic Roux-en-Y gastric bypass (RYGB) are used to resolve the problem of obesity in Saudi Arabia effectively [2]. The current endoscopic devices such as 
endoscopy gastroplasty with Apollo OverStitch Device, an FDA-approved device, that provide a more cost-effective and minimally invasive intervention are not widely accessible in the country [3]. Furthermore, according to American and European guidelines, a routine esophageal gastroduodenal endoscopy (EGD) is recommended for all candidates as part of the preoperative workup in morbidly obese patients who plan to undergo bariatric surgery [4,5]. However, it is still controversial whether or not to regularly screen obese patients before surgery. For all obese patients before bariatric procedures, several researchers suggested that an EGD be performed due to a lack of consistency between patient symptoms and outcomes. Also, they recommend routinely checking for Helicobacter pylori (H. pylori) and eradicating the bacteria before planning a bariatric procedure, although such data are limited [6]. However, due to the invasiveness and cost of EGD, some researchers recommend a selective strategy toward EGD for obese patients, as asymptomatic patients demonstrate poor clinical relevance concerning most of the lesions identified in a routine EGD $[7,8]$.

Unfortunately, there are limited and inconsistent data about abnormal endoscopic findings that may influence the management of obese patients undergoing bariatric surgery. Moreover, the prevalence of abnormal endoscopic findings in obese patients considered for bariatric surgery is reported to be insufficient compared to that of the non-obese population $[9,10]$. Additionally, the frequency of $H$. pylori infection in obese patients remains unclear.

This study aimed to report the patient characteristics, comorbid conditions, and symptoms at the time of preendoscopy, and to evaluate the prevalence of abnormal upper gastrointestinal (GI) endoscopic findings in obese Saudi patients before bariatric procedures. Moreover, we aimed to report the prevalence of $H$. pylori infection in the obese population and to record the potential risk factors for changing the surgery approach.

\section{Patients and methods}

The study was reviewed and approved by the Prince Sattam bin Abdulaziz Institutional Review Board (PASU/COM/ $\mathrm{RC} / \mathrm{A} / 45)$. Besides, the ethical approval of each hospital was obtained for database management.

${ }^{a}$ Endoscopy Unit, Department of Medicine, Prince Sattam bin Abdulaziz University Hospital, College of Medicine, Prince Sattam bin Abdulaziz University, Al Kharj (Georgios Zacharakis, Abdullah Altuwaim, Abdullah Bawazir, Ahmad Alonazi, Faisal Alsamari,

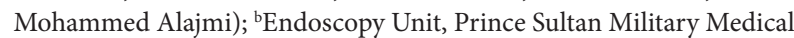
City, Riyadh (Abdulaziz Almasoud); ${ }^{c}$ Department of Family Medicine, College of Medicine, Prince Sattam bin Abdulaziz University, Al Kharj

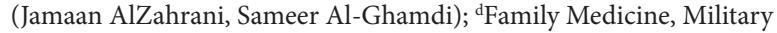
Hospital, Al Kharj (Abdullah AlShehri); ${ }^{\mathrm{e} B a r i a t r i c ~ C l i n i c, ~ K i n g ~ S a l m a n ~}$ Specialist Hospital, Hail (Ahmed Lotfy, Alexandros Kyritsis, Ioannis Terzis), ${ }^{\mathrm{f}}$ College of Computer and Information Sciences, Al-Imam Mohammad Ibn Saud Islamic University, Riyadh (Pavlos Nikolaidis), Saudi Arabia

\section{Study design and patients}

This was a retrospective study that reviewed the database of 1,129 patients who underwent bariatric surgery (LSG or RYGB) in Hail and Al-Kharj, Saudi Arabia, during the period between March 2018 and August of 2019. The data were collected in the bariatric clinics of King Salman Specialist Hospital in Hail, and Al-Kharj Military Hospital included only adult obese Saudi patients who fulfilled the criteria for surgery and who enrolled in the study once they provided informed consent. Regarding the assessment of patients, data was collected from the registry, database center medical records review, and clinical evaluations. Body mass index (BMI) was clinically measured.

Inclusion criteria were: Saudi patients aged 18-65 with a BMI $>35 \mathrm{~kg} / \mathrm{m}^{2}$; failure of many attempts of dietetic regimens; a record of comorbidities; and an acceptable level of surgical risk. Exclusion criteria were: evidence of non-compliance with the perioperative regimen or a prohibitive surgical risk; uncontrolled alcohol or drug dependence; uncontrolled depression or other psychiatric illnesses; and lack of family support or the existence of serious family discord about the proposed operation.

\section{Data collection and management}

We retrospectively checked the medical records of all morbidly obese patients before bariatric surgery that included characteristics of patients, comorbidities, and endoscopic and histological findings. In addition, we collected the GI symptoms, which included gastroesophageal reflux disease (GERD) symptoms such as heartburn, regurgitation, chest pain, dysphagia, and dyspepsia symptoms such as nausea with or without vomiting, epigastric pain or burning, bloating, and abdominal pain. All patients who underwent EGD had 3 endoscopic gastric biopsy specimens for the identification of $H$. pylori infection, 2 from the antrum, and 1 from the body. A histological identification based on normal hematoxylin and eosin stain was used to confirm the presence of $H$. pylori. The main indication for endoscopy was preoperative evaluation. Regarding the endoscopic finding's classification, we used the categorization of Sharaf et al, [11] who classified it to group 0 , group 1 , group 2, group 3 , and group 4.

\section{Statistical analysis}

We used a commercially available version of Statistical Package of Social Science (SPSS version 22 for Windows; SPSS Inc, Chicago, IL). Continuous data were presented as mean and standard deviation (SD) and were compared using a Student's $t$-test or a nonparametric test. Categorical variables were presented in frequency and percentage and were analyzed using the $\chi^{2}$ or Fisher's exact tests. Analysis 
of the logistic regression was used to define preoperative predictors that could be associated with abnormal endoscopic outcomes. A 2-tailed $\mathrm{P}<0.05$ was considered to be statistically significant.

\section{Results}

\section{Demographics and clinical characteristics}

Of the 717 patients who underwent EGDs, 432 underwent bariatric surgery. The majority of the performed bariatric procedures were LSG 412 (95.37\%), followed by RYGB 20 (4.62\%). The mean BMI was $44.3 \pm 6.3 \mathrm{~kg} / \mathrm{m}^{2}$, and the mean age was $27.8 \pm 11.8$ years old. The predominant gender was female (65\%). Overall, $38 \%$ had one or more comorbid diseases. Females had a higher prevalence of any comorbidity $(65.8 \%$ vs. $57.8 \%)$ compared with males. Those with more comorbidities were significantly older $(\mathrm{P}=0.004)$. There was a significant difference in age groups between those without comorbidities (23.3 years, 95\% confidence interval [CI] 32.8-34.1), vs. those with 1 comorbidity (27 years, 95\%CI $32.7-35.6$ ), and those with more than 2 comorbidities (36 years, 95\%CI 34.9-36.7). Regarding upper GI symptoms (UGIS), the overall prevalence was 38\%. The prevalence of GERD symptoms was 387 (54\%), followed by dyspepsia symptoms, which were present in 315 (44\%) obese patients considered for bariatric surgery. The most commonly reported comorbidity, in over half of the patients, was diabetes mellitus type II (57\%), followed by hypertension (50\%), osteoarthritis (35\%), hypothyroidism (34\%), and chronic back pain (33\%). Depression and anxiety were less frequently reported (11\%). Obesity-related comorbidities are shown in Table 1.

\section{EGD findings}

All the 717 obese Saudi patients were routinely evaluated with a preoperative EGD, including a biopsy, in the dedicated bariatric centers before bariatric surgery, as shown in Table 2. Preoperative EGD revealed abnormalities in 459 (64\%) patients. The most common endoscopic finding was hiatal hernia ( $\mathrm{HH})$ in 308 (43\%) patients, followed by gastritis (42\%), and esophagitis (14.4\%). Pre-bariatric EGD showed $\mathrm{HH}$ with the presence of esophagitis and GERD symptoms in 64 (14.8\%) of the 432 patients who underwent bariatric surgery. Of those patients, 30 (9.7\%) underwent LSG; GERD symptoms improved in $21(70 \%)$ and worsened in the other $9(30 \%)$.

The total patients in group 0 with normal EGD were 258 (36\%) of the 717 who underwent EGD. These patients were either on the waiting list or underwent bariatric surgery without a delay or modified procedure.

In group 1, 367 (51.2\%) patients of the 717 who underwent EGD had abnormal EGD findings such as mild esophagitis, gastritis, duodenitis, and esophageal web. However, of those who planned to undergo bariatric surgery in group 1, 138
Table 1 Obesity-related comorbidities

\begin{tabular}{llcc}
\hline Characteristics & & $\mathrm{N}$ & $\%$ \\
\hline $\begin{array}{l}\text { Upper gastrointestinal } \\
\text { symptoms }\end{array}$ & GERD & 387 & 54 \\
& Dyspepsia & 315 & 44 \\
& Dysphagia & 15 & 2 \\
Cardiovascular disease & Hypertension & 361 & 50 \\
& Coronary artery disease & 65 & 9.1 \\
\hline Pulmonary diseases & Asthma/COPD & 57 & 8 \\
Endocrine diseases & Diabetes mellitus & 408 & 57 \\
& Hypothyroidism & 243 & 34 \\
\hline Mental diseases & Depression/anxiety & 79 & 11 \\
\hline Bone diseases & Osteoarthritis & 250 & 35 \\
& chronic back pain & 236 & 33 \\
\hline Other functional disorders & Abdominal pain, IBS-like & 82 & 11.4 \\
\hline Other diseases & Anemia & 19 & 2.6 \\
& Cancer & 15 & 2.1 \\
\hline
\end{tabular}

GERD, gastroesophageal reflux disease; COPD, chronic obstructive pulmonary disease; IBS, irritable bowel syndrome

Table 2 Abnormal findings from EGD of enrolled patients before bariatric procedure

\begin{tabular}{llcc}
\hline \multirow{2}{*}{ Abnormal EGD findings* } & $\mathrm{N}$ & $\%$ \\
\hline Esophagus & $\mathrm{HH}$ & 308 & 43 \\
& Large $\mathrm{HH}>4 \mathrm{~cm}$ & 27 & 3.8 \\
& Esophagitis & 103 & 14.4 \\
& LA classification: A/B/C/D & $59 / 37 / 6 / 1$ & $57.3 / 36 / 5.8 / 0.9$ \\
& Barrett's esophagus & 3 & 0.4 \\
& Varices & 7 & 0.8 \\
Stomach & Atrophic gastritis & 8 & 1.2 \\
& Erosive gastritis & 64 & 8.9 \\
& H. pylori $(+)$ gastritis & 286 & 42.1 \\
& Ulcers & 9 & 1.25 \\
& Polyps/large $>1 \mathrm{~cm}$ & $56 / 10$ & $7.8 / 1.4$ \\
Duodenum & Duodenitis & 29 & 4 \\
& Ulcers & 16 & 2.2 \\
\hline Cancer & & 2 & 0.7 \\
\hline Total & & 459 & 64 \\
\hline
\end{tabular}

ॠAlmost a third reported had more than one endoscopic abnormal finding

HH, hiatal hernia; LA, The Los Angeles Classification;

EGD, esophagogastroduodenoscopy; H. pylori, Helicobacter pylori

(31.9\%) of 432 neither changed the surgical approach nor postponed it because of these endoscopic findings.

Patients with delayed bariatric procedure had: large polyps of $>1 \mathrm{~cm}$ (either hyperplastic or cystic polyps) 10 (2.3\%); esophagitis Los Angeles class C/D 7 (1.62\%); Barrett's esophagus $3(0.7 \%)$; and peptic ulcer disease $25(5.7 \%)$. The total number 
of patients in group 2 in whom the bariatric procedure was changed or delayed (due to mass mucosal/submucosal lesions, peptic ulcer disease, severe erosive esophagitis, Barrett's esophagus, $\mathrm{HH}$ of any size, peptic stricture, Zenker's diverticula, and arteriovenous malformations) were 65 (15.04\%).

A modified bariatric procedure was carried out in 30 of 64 (47\%) patients with medium-sized and large $\mathrm{HH}$ with GERD symptoms and esophagitis. They underwent RYGB, while the rest refused to change the bariatric procedure and underwent LSG, the most popular bariatric procedure in Saudi Arabia. Peptic ulcer operation postponed the bariatric surgery and medications prescribed until a follow-up endoscopy confirmed full healing.

A bariatric procedure was contraindicated and thus postponed in patients with varices $7(0.97 \%)$ and malignant lesions $2(0.27 \%)$. The total number of patients in group 3 with absolute contraindications for surgery, such as upper GI cancer or varices, was 9 (1.26\%). H. pylori infection was assessed during the histopathological examinations of $672(42.4 \%)$ patients; 287 were tested positive. There was a significant variation in $H$. pylori infection in obese patients between AlKharj and Hail hospitals 102/147 (69\%) vs. 185/525 (35\%), $\mathrm{P}<0.001$, respectively (Fig. 1,2).

The mean age for $H$. pylori-positive patients was $39.6 \pm 12.03$ years, while the mean age for $H$. pylori-negative patients was $31.3 \pm 9.3$ years $(\mathrm{P}=0.01)$. Older people were more likely to have H. pylori infection. In obese patients at Al-Kharj, 43 (29.5\%) males and $104(70.1 \%)$ females were $H$. pylori-positive, while among those at Hail, 69 (37.3\%) males and 116 (62.7\%) females were $H$. pylori-positive. $H$. pylori infection was more prevalent in Hail $(\mathrm{P}<0.001)$, and there was not a significant difference between males and females between the 2 cities $(\mathrm{P}=0.0587)$. Fig. 3 presents the change of bariatric surgery based on preendoscopy findings.

\section{Discussion}

The objective of this study was to evaluate the rate of comorbidities related to obesity in the Saudi population. Nonetheless, this analysis offers an approximate estimation of the frequency of comorbidities attributed to obese Saudis. The most commonly reported comorbidity, in over half of patients, was diabetes mellitus type II, followed by hypertension and osteoarthritis. Depression and anxiety were less frequently reported. The most frequent UGIS in obese patients considered for bariatric surgery were GERD and dyspepsia symptoms. Women were more likely to report a comorbidity. Our results indicate that obesity is a significant health burden.

Few studies worldwide have shown a high prevalence of H. pylori ranging between $11-39 \%$ in Europe and $61 \%$ in USA in morbidly obese patients undergoing bariatric surgery [12-17]. Also, few studies looked only at the $H$. pylori serologies, which might not reflect an active infection [17]. This study showed an exceptionally high prevalence of the infection in an unselected group of morbidly obese patients in a relatively young age group who have undergone EGD before weight reduction surgeries. The explanation of such a high prevalence is not clear and has not

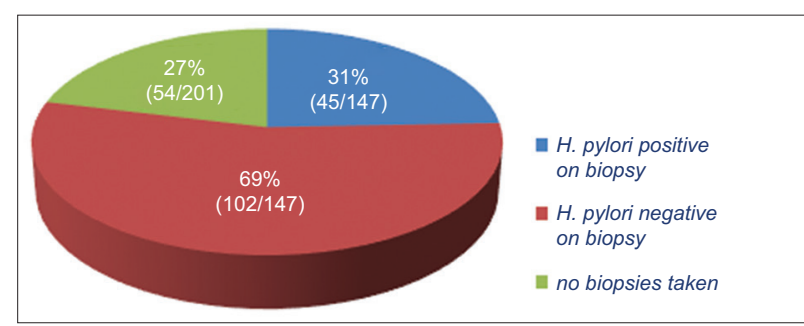

Figure 1 Prevalence of Helicobacter pylori (H. pylori) among bariatric patients in Al-Kharj

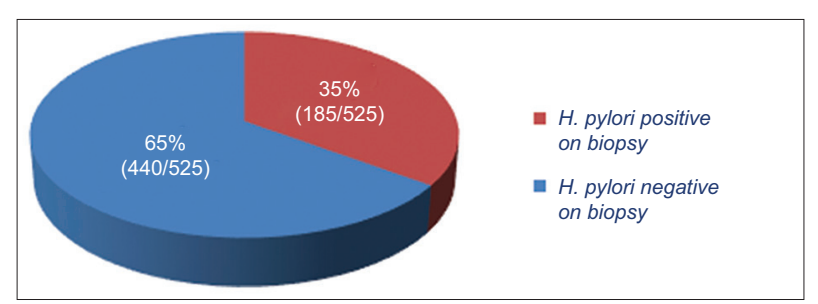

Figure 2 Prevalence of Helicobacter pylori (H. pylori) among bariatric patients in Hail

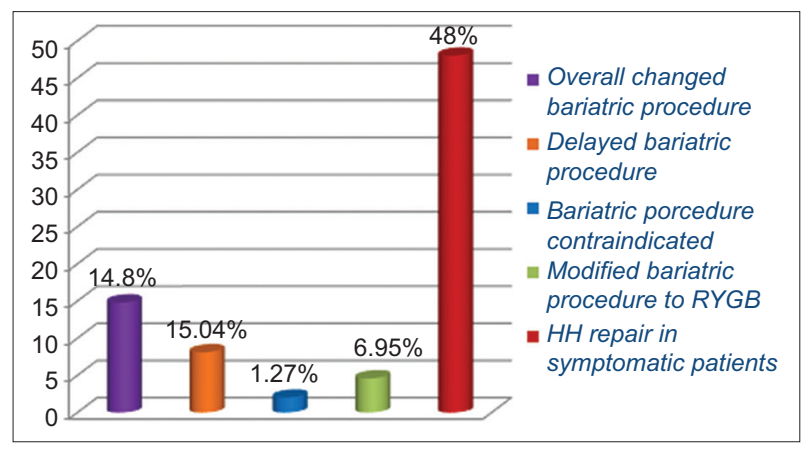

Figure 3 Change of bariatric surgery based on pre-endoscopy findings HH, hiatal hernia; RYGB, Roux-en-Y gastric bypass

been clarified. In our study, about two-thirds of obese individuals were infected with $H$. pylori in Al Kharj, but the rate was only onethird in Hail $(\mathrm{P}<0.001)$. Finally, the frequency of $H$. pylori was significantly higher in obese females in both Hail and Al-Kharj compared to males $(\mathrm{P}=0.002)$. In patients with different biopsyproven GI conditions, such as gastritis, peptic ulcer disease, and gastric cancer, the prevalence of $H$. pylori in Saudi Arabia was $71.33 \%$, with a slight female predominance [18].

In a recent study in Al-Kharj [19], a total of 474 Saudi patients with functional dyspepsia according to the ROME IV criteria were screened for $H$. pylori infection through the use of the urea breath test. The study showed a high prevalence of $H$. pylori infection in 182 of 474 (38.5\%) patients with dyspepsia living in the central rural region of Saudi Arabia. The frequency of $H$. pylori infection among females in the AlKharj region was estimated as 102 of 164 (62.2\%) compared to 80 of $128(62.5 \%)$ in males $(\mathrm{P}=0.63)$. The majority of the $H$. pylori-infected patients were in the age group 22-44 years old (67.9\%). Another study by Aleid et al [20] demonstrated that the prevalence of $H$. pylori in patients assigned to upper GI endoscopy following bariatric surgery in Riyadh was 39\%. This 
finding was quite similar to our Hail's patients (35\%) but much lower than Al-Kharj (69\%). This heterogeneity among Saudi Arabia regions is due to several factors, including personal hygiene, healthcare system, and socioeconomic status.

In a group of morbidly obese Saudi patients with chronic active gastritis, the prevalence of $H$. pylori infection was high (85.5\%). Interestingly, the prevalence of $H$. pylori infection was similar in patients with and without comorbid conditions. The main endoscopic findings were gastric erosions in $13 \%, \mathrm{HH}$ in $13 \%$, and gastritis in $67.7 \%$, with no records of duodenal or gastric ulcers [21].

Our study showed that one-third of the patients had normal EGD findings following successful bariatric surgeries without delay or a modified procedure. Almost half of the patients had abnormal EGD findings; however, these findings were mild. Only $15 \%$ delayed the decision plan for bariatric surgery. Patients with $\mathrm{HH}$ were subjected to modified surgery to repair and reduce the hernia. Esophagitis, gastric ulcers, duodenal ulcers, and polyps led to the postponement of the operation. Medications were administered after a follow-up endoscopy confirmed complete recovery. Abnormal findings of EGD in those patients with UGIS were high. A recent systematic review of 48 studies showed that the proportion of EGDs resulting in a change in surgical management was 7.8\%. Of them, 221 (44.9\%) were $\mathrm{HH}$ repairs, and $201(40.8 \%)$ were delays in surgery due to gastritis or peptic ulcer disease. Moreover, 37 (7.5\%) patients required major changes in the planned procedure, such as switching from RYGB to LSG or adding a gastrectomy to a RYGB, and $4(0.8 \%)$ were cancellations due to esophageal cancer or varices [22]. Similarly, another meta-analysis of 28 studies demonstrated that $7.6 \%$ of the enrolled patients had findings that delayed/altered surgery. Moreover, they showed that the most common findings were gastritis, $\mathrm{HH}, \mathrm{H}$. pylori, and esophagitis [23].

The main limitation of this observational study on the role of routine preoperatively EGD in bariatric patients was the retrospective design, which may aggravate the risk of recall bias. We could not compare the types of bariatric surgery in terms of EGDs due to the unequal distribution of our sample. Another limitation was the predominance of females in our population. Finally, it is very important that current endoscopic treatments for obesity such as endoscopic gastroplasty with Apollo OverStitch Device, which is non-invasive compared to surgical sleeve gastrectomy, is not accessible in our centers as in the majority of bariatic centers across the country. Therefore, data on the role of routine EGD before endoscopic gastroplasty is not available although its role is equally important to preoperative EGD.

In conclusion, GERD and $\mathrm{HH}$ are highly prevalent in obese patients. Variable rates of $H$. pylori infection in the bariatric population were recorded throughout Saudi Arabia. Endoscopic and histologic findings were shared in preoperative EGDs but did not significantly change the planned surgical procedure. Routine, preoperative EGD assessment in the obese Saudi population is obviously mandatory not only to identify the specific individual anatomy, but also to exclude early asymptomatic lesions or even early gastric cancer. A campaign should be developed to increase the awareness of Saudi populations towards the complications of LSG.

\section{Summary Box}

\section{What is already known:}

- A routine preoperative esophagogastroduodenos copy (EGD) is recommended for all morbidly obese patients who plan to undergo bariatric surgery although some researchers recommend a selective strategy toward EGD for obese patients

- Routinely screening for Helicobacter pylori (H. pylori) and eradicating it before planning a bariatric procedure is recommended, although such data are limited

- There are limited and inconsistent data about abnormal endoscopic findings that may influence the management of obese patients undergoing bariatric surgery

\section{What the new findings are:}

- Routine, preoperative EGD assessment in the obese Saudi population is mandatory not only to identify the specific individual anatomy, but also to exclude early asymptomatic lesions or even early gastric cancer

- Our findings (demographic data, comorbid conditions, symptoms) confirmed that obesity carries a profound health burden in Saudi Arabia

- Variable rates of H. pylori infection in the bariatric population were recorded throughout Saudi Arabia

\section{Acknowledgment}

This project was supported by Prince Sattam bin Abdulaziz University, Deanship of Scientific Research, College of Medicine

\section{References}

1. Alnohair S. Obesity in Gulf Countries. Int J Health Sci (Qassim) 2014;8:79-83.

2. El Chaar M, Hammoud N, Ezeji G, et al. Laparoscopic sleeve gastrectomy versus laparoscopic Roux-en-Y gastric bypass: a single center experience with 2 years follow-up. Obes Surg 2015;25:254-262.

3. Sampath K, Dinani AM, Rothstein RI. Endoscopic devices for obesity. Curr Obes Rep 2016;5:251-261.

4. Sauerland S, Angrisani L, Belachew M, et al. Obesity surgery: Evidence-based guidelines of the European Association for Endoscopic Surgery (EAES). Surg Endosc 2005;19:200-221.

5. Anderson MA, Gan SI, Fanelli RD, et al. Role of endoscopy in the bariatric surgery patient. Gastrointest Endosc 2008;68:1-10.

6. Pintar T, Kaliterna N, Carli T. The need for a patient-tailored Helicobacter pylori eradication protocol prior to bariatric surgery. $J$ 
Int Med Res 2018;46:2696-707.

7. Peromaa-Haavisto P, Victorzon M. Is routine preoperative upper GI endoscopy needed prior to gastric bypass? Obes Surg 2013;23:736739 .

8. Loewen M, Giovanni J, Barba C. Screening endoscopy before bariatric surgery: a series of 448 patients. Surg Obes Relat Dis 2008;4:709-712.

9. Frigg A, Peterli R, Zynamon A, Lang C, Tondelli P. Radiologic and endoscopic evaluation for laparoscopic adjustable gastric banding: preoperative and follow-up. Obes Surg 2001;11:594-599.

10. Korenkov M, Köhler L, Yücel N, et al. Esophageal motility and reflux symptoms before and after bariatric surgery. Obes Surg 2002; 12:72-76.

11. Sharaf RN, Weinshel EH, Bini EJ, Rosenberg J, Sherman A, Ren CJ. Endoscopy plays an important preoperative role in bariatric surgery. Obes Surg 2004;14:1367-1372.

12. Renshaw AA, Rabaza JR, Gonzalez AM, Verdeja JC. Helicobacter pylori infection in patients undergoing gastric bypass surgery for morbid obesity. Obes Surg 2001;11:281-283.

13. Ramaswamy A, Lin E, Ramsaw BJ, Smith CD. Early effects of Helicobacter pylori infection in patients undergoing bariatric surgery. Arch Surg 2004;139:1094-1096.

14. Papasavas PK, Gange DJ, Donnelly PE, et al. Prevalence of Helicobacter pylori infection and value of preoperative testing and treatment in patients undergoing laparoscopic Roux-en-Y gastric bypass. Surg Obes Relat Dis 2008;4:383-388.

15. Vanek VW, Catania M, Triveri K, Woodruff RW Jr. Retrospective review of preoperative biliary and gastrointestinal evaluation for gastric bypass surgery. Surg Obes Relat Dis 2006;2:17-22.

16. de Moura Almeida A, Cotrim HP, Santos AS, et al. Preoperative upper gastrointestinal endoscopy in obese patients undergoing bariatric surgery: Is it necessary? Surg Obes Relat Dis 2008;4:144-149.

17. Erim T, Cruz-Correa MR, Szomstein S, Velis E, Rosenthal R. Prevalence of Helicobacter pylori seropositivity among patients undergoing bariatric surgery: A preliminary study. World J Surg 2008;32:2021-2025.

18. Alhussaini MS. Prevalence of Helicobacter pylori among patients with different gastrointestinal disorders in Saudi Arabia. Med J Indones 2017;25:214-220.

19. Alanazi BG, Alanazi FH, Albriek AZ, et al. The prevalence of Helicobacter pylori infection in patients with dyspepsia in the central rural region of Saudi Arabia. Indo Am J P Sci 2019;6:1358-1364.

20. Aleid A, Al Balkhi A, Hummedi A, et al. The utility of esophagogastroduodenoscopy and Helicobacter pylori screening in the preoperative assessment of patients undergoing bariatric surgery: A cross-sectional, single-center study in Saudi Arabia. Saudi J Gastroenterol 2020;26:32.

21. Al-Akwaa A. Prevalence of Helicobacter pylori infection in a group of morbidly obese Saudi patients undergoing bariatric surgery: A preliminary report. Saudi J Gastroenterol 2010;16:264.

22. Bennett S, Gostimir M, Shorr R, et al. The role of routine preoperative upper endoscopy in bariatric surgery: a systematic review and meta-analysis. Surg Obes Relat Dis 2016;12:1116-1125.

23. Parikh M, Liu J, Vieira D, et al. Preoperative endoscopy prior to bariatric surgery: a systematic review and meta-analysis of the literature. Obes Surg 2016;26:2961-2966. 